\title{
Identification of stress resilience module by weighted gene co-expression network analysis in Fkbp5-deficient mice
}

\author{
Joonhong Kwon, Yeong Jae Kim, Koeul Choi, Sihwan Seol and Hyo Jung Kang ${ }^{*}$ (B)
}

\begin{abstract}
FKBP5 encodes the FK506 binding protein 5, a glucocorticoid receptor (GR) binding protein known to play an important role in the physiological stress response. However, results from previous studies examining the association between common variants of FKBP5 and stress have been inconsistent. To investigate whether the loss of FKBP5 affects the stress response, we examined the behavior of mice following the induction of chronic restraint stress between homozygous wild-type and Fkbp5 knock-out mice. After 21 days of exposure to restraint stress, WT mice showed anhedonia, a core symptom of depression, which could be measured by a sucrose preference test. However, Fkbp5deficient mice did not exhibit significant depressive-like behavior compared to the WT after exposure to chronic restraint stress. To investigate the molecular mechanism underlying stress resilience, we performed RNA sequencing analysis. The differentially expressed gene (DEG) analysis showed that chronic stress induced changes in various biological processes involved in cell-cell adhesion and inflammatory response. Weighted gene co-expression network analysis identified 60 characteristic modules that correlated with stress or the FKBP5 genotype. Among them, M55 showed a gene expression pattern consistent with behavioral changes after stress exposure, and the gene ontology analysis revealed that this was involved in nervous system development, gland morphogenesis, and inflammatory response. These results suggest that FKBP5 may be a crucial factor for the stress response, and that transcriptomic data can provide insight into stress-related pathophysiology.
\end{abstract}

Keywords: FKBP5, Chronic stress, Resilience, RNAseq, WGCNA

\section{Main text}

Depression is one of the most common mental disorders affecting people of all ages, and can arise from a variety of causes, including genetic susceptibility, endocrine dysregulation, and stresses in life [1]. When exposed to acute and temporary stress, while the body can protect itself from stress, chronic stress can disturb the function of the brain system. Chronic accumulation of stress leads to abnormal and excessive cortisol secretion in the hypothalamic-pituitary-adrenal axis, which affects a variety of physical activities, including brain function, leading to mental disorders such as depression or post-traumatic stress disorder (PTSD) [2]. Proper regulation through negative feedback of glucocorticoid receptors (GR) is important for the stress response, and long-term or excessive

\footnotetext{
* Correspondence: hyokang@cau.ac.kr

Department of Life Science, Chung-Ang University, 84 Heukseok-ro, Dongjak-gu, Seoul 06974, South Korea
}

activation of this system is associated with the development of depression or anxiety disorders [3]. The FK506binding protein 51 (FKBP5) is a co-chaperone of Hsp90 in the GR molecular complex and is a key modulator of GR sensitivity $[4,5]$. Although FKBP5 is an important factor that is responsible for coping behavior as well as neuroendocrine responses [6], results from previous studies investigating the association between $F k b p 5$ gene variants and stress remain controversial. In order to investigate whether genetic FKBP5 variants affect behavior in response to chronic restraint stress exposure, we examined the behavior of mice following the induction of chronic restraint stress in homozygous wild-type (WT) and knock-out (KO) mice. After 21 days of exposure to restraint stress, while WT mice showed anhedonia in the sucrose preference test, $F k b p 5$-deficient mice did not exhibit significant depressive-like behavior compared to the WT (Fig. 1)a and b.

(c) The Author(s). 2019 Open Access This article is distributed under the terms of the Creative Commons Attribution 4.0 International License (http://creativecommons.org/licenses/by/4.0/), which permits unrestricted use, distribution, and reproduction in any medium, provided you give appropriate credit to the original author(s) and the source, provide a link to the Creative Commons license, and indicate if changes were made. The Creative Commons Public Domain Dedication waiver (http://creativecommons.org/publicdomain/zero/1.0/) applies to the data made available in this article, unless otherwise stated. 


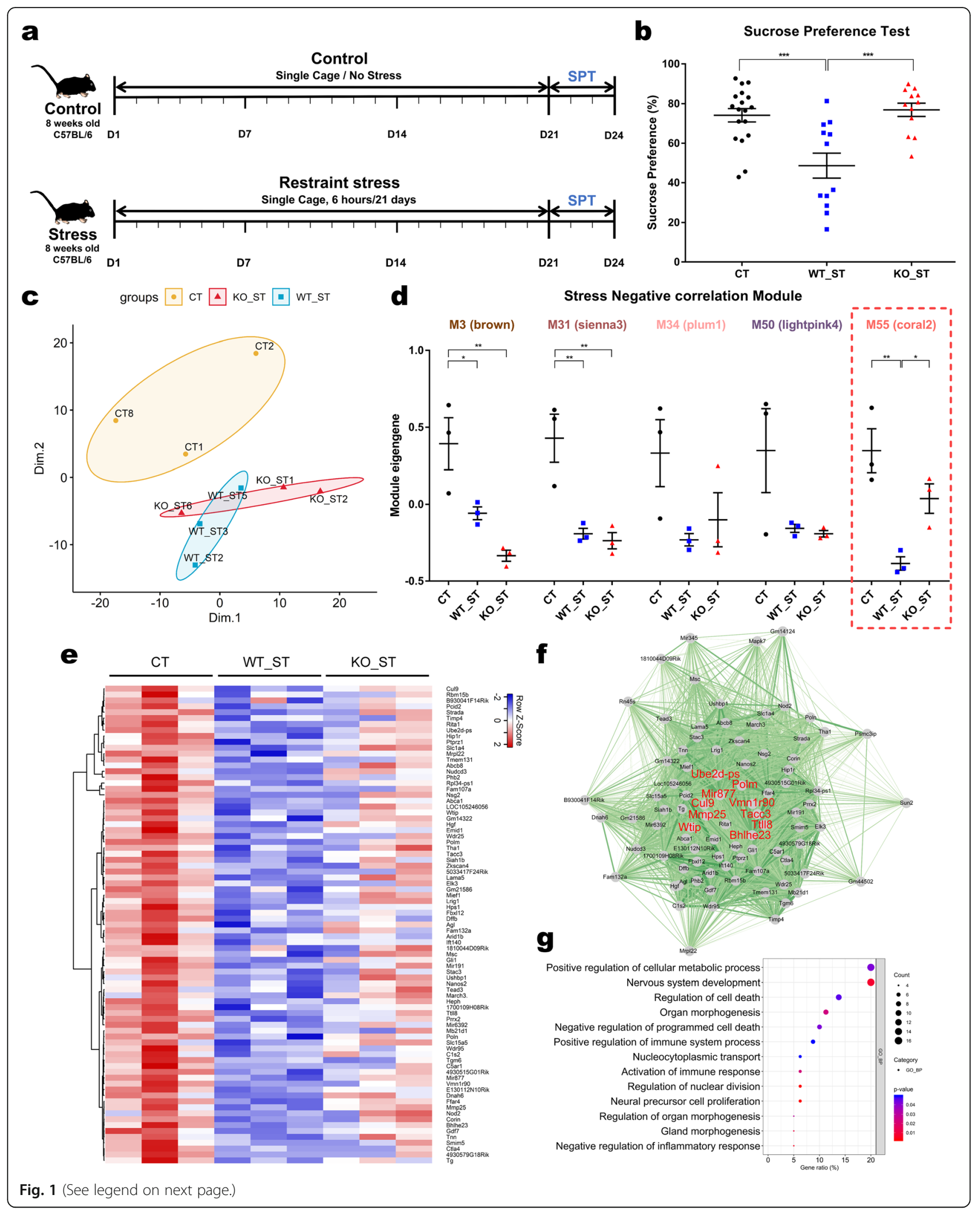


(See figure on previous page.)

Fig. 1 Transcriptome analyses of the mPFC in homozygous wild-type (WT) and Fkbp5 knock-out (KO) mice following chronic stress. a Schematic timeline of the induction of chronic restraint stress. $\mathbf{b}$ Effect of CRS on sucrose preference. Data were combined with the 1st and 2nd sucrose preference test (SPT) results. Control mice (CT) $n=9$; CRS-exposed WT mice (WT_ST) $n=6$; CRS-exposed Fkbp5 KO mice (KO_ST) $n=6$. One-way ANOVA $(F[2,39]=11.67, p=0.0001)$; Fisher's LSD: ${ }^{* *} p<0.001$. c Multidimensional scaling (MDS) plot for transcriptomes of individual samples of CT (yellow), WT_ST (blue) or KO_ST (red). d The interleaved scatter plots of modules which have a significant negative correlation with stress. Data represent mean \pm SEM. One-way ANOVA; Fisher's LSD: ${ }^{*} p \leq 0.05,{ }^{* *} p<0.01$. e A heatmap showing the expression of M55 genes in the mPFC of CT (left), WT_ST (middle) and KO_ST (right). f A network plot of M55 genes and their intramodular connections. The ten hub genes (the top ten genes with highest intramodular connectivity; Cul9, Polm, Ttll8, Vmn1r90, Tacc3, Mir877, Mmp25, Bhlhe23, Wtip and Ube2d-ps) are shown in red. Note their central position in the network, suggesting high intramodular connectivity. g Enrichment dot plot for Gene Ontology (GO) analysis of M55 genes. The $13 \mathrm{GO}$ terms with the lowest $p$-value each annotation cluster are plotted in order of gene ratio. The dot size represents the number of genes associated with a specific term. The dot color represents the adjusted p-value

Recent studies performed in humans and rodents have suggested that long-term stress and pathological anxiety leads to structural degeneration and functional alteration of the frontal cortex, and increases the risk of mental disorders $[7,8]$. In addition, it has been suggested that the medial prefrontal cortex (mPFC), a region controlling higher brain function including cognition and emotion, is a primary target of stress hormones $[9,10]$. However, little is known about the molecular mechanisms in the mPFC involved in stress-associated psychiatric disorders. Transcriptome profiling has helped provide an unbiased insight into the pathophysiological mechanism underlying complicated brain disorders [11]. Therefore, using RNA sequencing (RNAseq) analysis, we investigated the dynamic transcriptomic changes that occur after stress in the mPFC of Fkbp5-deficient mice.

Multidimensional scaling analysis showed a clear separation between the stressed and the non-stressed control (CT) mice. There was also a slight overlap between the CRS-exposed WT mice (WT_ST) and CRS-exposed Fkbp5 KO mice (KO_ST) groups, which were clustered according to their genotype (Fig. 1)c. To identify the genotypes and genes affected by stress, we analyzed differentially expressed genes (DEG) between each group (Additional file 1). Of the 24,532 mRNA genes profiled, 224 (0.91\%; CT vs WT_ST), 258 (1.05\%; CT vs KO_ST), and 135 (0.55\%; WT_ST vs KO_ST) genes were dysregulated in the $\mathrm{mPFC}$ following chronic restraint stress, and the percentage of DEG induced by stress was higher than the percentage of DEG by genotype (Additional file 2: Figure S1, Additional file 3: Table S1-S3). Gene ontology (GO) enrichment analysis of these expression profiles showed functional categories that were potentially dysregulated, including lipid metabolic process (Benjamini adjusted $\left.p=9.63 \times 10^{-4}\right)$, regulation of immune response (Benjamini adjusted $p=7.24 \times 10^{-4}$ ), cell adhesion (Benjamini adjusted $p=1.52 \times 10^{-3}$ ), regulation of cell differentiation (Benjamini adjusted $p=1.17 \times 10^{-3}$ ), and neurogenesis (Benjamini adjusted $p=7.66 \times 10^{-2}$ ) (Additional file 4: Table S4-S6).

To compare the differences in expression observed in the multidimensional data set with the pattern of stress- response behavior, we performed a weighted gene coexpression network analysis (WGCNA) [12]. Through WGCNA, we identified 60 modules of co-expressed genes following chronic restraint stress in both WT and KO homozygous genotypes (Additional file 5: Figure S2, Additional file 6: Table S7). Among the 60 modules, we identified characteristic modules which showed a significant correlation to the genotype (M3, M6, M25, M33, M39, M44 and M57) and to the stress exposure (M3, M10, M18, M21, M31, M34, M50 and M55) (Fig. 1, Additional file 7: Figure S3)d, a-c. Interestingly, one of the modules that negatively correlated with stress, M55, had a pattern similar to the stress resilience behavior of Fkbp5-deficient mice. This was down-regulated, consistent with depression-like behavior in the WT_ST group, and was restored in the KO_ST group (Fig. 1)d and e. GO enrichment analysis of M55 revealed the biological functions potentially involved, including gland morphogenesis $\left(p=1.25 \times 10^{-2}\right)$, activation of immune response $\left(p=2.25 \times 10^{-2}\right)$, and nervous system development $(p=$ $7.65 \times 10^{-3}$ ) (Fig. 1, Additional file 8: Table S8)f and g. Hub genes, with the highest degree of connectivity within a module of the M55 include Mmp25. This gene has been functionally implicated in the regulation of immune response through NF-B signaling [13] and has been linked to neuropsychiatric disorders including PTSD [14].

In this study, we compared brain transcriptome altered by chronic stress in the mPFC between Fkbp5-deficient and wild-type mice by RNAseq analysis. In addition to the DEG analysis, by employing WGCNA, we identified a distinct co-expression network module associated with stress resilience caused by $F k b p 5$ knock-out, and characterized the biological processes affected by this module, leading to this unique behavior. Our systematic transcriptome analysis demonstrated that aberration in the development of the neuroendocrine system, and regulation of the immune response may underlie the stress resilient behavior observed in the $F k b p 5$ deficient mice. This is the first study, to the best of our knowledge, to identify the stress resilience associated genes through gene co-expression network analysis in $F k b p 5$ deficient mice. Altogether, we 
confirmed that FKBP5 may be an important component of the stress response, suggesting that identification of the module associated with the stress response can provide a treatment strategy and therapeutic target to attenuate the depressive symptoms caused by stress.

\section{Supplementary information}

Supplementary information accompanies this paper at https://doi.org/10. 1186/s13041-019-0521-9.

\section{Additional file 1. Materials and Methods.}

Additional file 2: Figure S1. Heatmap representing the expression profiles of the DEGs in the three groups of CT, WT_ST, and KO_ST.

Additional file 3: Table S1. List of DEG between WT ST and CT. Table S2. List of DEG between KO ST and CT. Table S3. List of DEG between KO ST and WT ST.

Additional file 4: Table S4. GO analysis for DEGs between WT ST and CT. Table S5. GO analysis for DEGs between KO ST and CT. Table S6. GO analysis for DEGs between KO ST and WT ST.

Additional file 5: Figure S2. Heatmap of the correlation of WGCNA modules with traits. The correlation between each module eigengene and sample trait was calculated. Values in the figure indicate the correlation coefficient between modules and traits. Values in brackets are the $p$-values for the association test.

Additional file 6: Table S7. List of genes within each WGCNA module (kME > 0.7).

Additional file 7: Figure S3. The interleaved scatter plots of module which have a significant correlation with genotype and stress (a-b) Modules with positively (a) and negatively (b) correlated to the genotype. (c) Modules with positively correlated to the stress. Data represent mean \pm SEM. One-way ANOVA; Fisher's LSD: ${ }^{*} p \leq 0.05$, ${ }^{* *} p<0.01$, *** $p<0.001$.

Additional file 8: Table S8. GO analysis for genes in M55.

\section{Abbreviations}

bh.pval: Benjamini-Hochberg adjusted P-value; CRS: Chronic restraint stress; CT: Control; DEG: Differentially expressed genes; GO: Gene ontology; KO: Knock-out; KO_ST: CRS-exposed Fkbp5 KO mice; mPFC: Medial prefrontal cortex; PTSD: Post-traumatic stress disorder; RNAseq: RNA sequencing; WGCNA: Weighted gene co-expression network analysis; WT: Wild-type; WT_ST: CRS-exposed WT mice

\section{Acknowledgements}

Not applicable.

\section{Authors' contributions}

JK, KC, SS performed molecular experiments and analysis of the sequencing data. YK was involved in the acquisition of animal behavior data. JK, YK drafted the manuscript. HJK designed the study and interpreted the results. HJK wrote the manuscript and all authors read and approved the final version of the manuscript.

\section{Funding}

This research was supported by the Chung-Ang University Scholarship Grants in 2016 and the Brain Research Program of the National Research Foundation of Korea (NRF) funded by the Ministry of Science and ICT (NRF-2018M3C7A1024150).

\section{Availability of data and materials}

The datasets supporting the conclusions of this article are included in this published article and its additional files. RNAseq raw data reported in this paper are submitted to the GEO repository under the accession number GSE138240.

\section{Ethics approval}

The animal experiments were conducted in accordance with the Chung-Ang University Council Directive for the use and care of laboratory animals, and
National Institutes of Health animal care guidelines. The Institutional Animal Care and Use Committee of Chung-Ang University approved all protocols described in this study.

Consent for publication

Not applicable.

\section{Competing interests}

The authors declare that they have no competing interests.

Received: 28 September 2019 Accepted: 13 November 2019 Published online: 27 November 2019

\section{References}

1. Jabbi M, Korf J, Kema IP, Hartman C, van der Pompe G, Minderaa RB, et al. Convergent genetic modulation of the endocrine stress response involves polymorphic variations of 5-HTT. COMT MAOA Mol Psychiatry. 2007:12:483.

2. de Kloet CS, Vermetten E, Geuze E, Kavelaars A, Heijnen CJ, Westenberg HG. Assessment of HPA-axis function in posttraumatic stress disorder: pharmacological and non-pharmacological challenge tests, a review. J Psychiatr Res. 2006;40:550.

3. Lucassen PJ, Pruessner J, Sousa N, Almeida OF, Van Dam AM, Rajkowska G, et al. Neuropathology of stress. Acta Neuropathol. 2014;127:109.

4. Wiederrecht G, Hung S, Chan HK, Marcy A, Martin M, Calaycay J, et al. Characterization of high molecular weight FK-506 binding activities reveals a novel FK-506-binding protein as well as a protein complex. J Biol Chem. 1992;267:21753.

5. Zannas AS, Wiechmann T, Gassen NC, Binder EB. Gene-stress-epigenetic regulation of FKBP5: clinical and translational implications. Neuropsychopharmacol. 2016:41:261.

6. Touma C, Gassen NC, Herrmann L, Cheung-Flynn J, Bull DR, lonescu IA, et al FK506 binding protein 5 shapes stress responsiveness: modulation of neuroendocrine reactivity and coping behavior. Biol Psychiatry. 2011;70:928.

7. Korgaonkar MS, Fornito A, Williams LM, Grieve SM. Abnormal structural networks characterize major depressive disorder: a connectome analysis. Biol Psychiatry. 2014;76:567.

8. Laine MA, Sokolowska E, Dudek M, Callan SA, Hyytia P, Hovatta I. Brain activation induced by chronic psychosocial stress in mice. Sci Rep. 2017;7:15061.

9. Arnsten AF. Stress signalling pathways that impair prefrontal cortex structure and function. Nat Rev Neurosci. 2009;10:410.

10. Broadbent DE. Decis Stress. London: Academic; 1971

11. Girgenti MJ, Duman RS. Transcriptome alterations in posttraumatic stress disorder. Biol Psychiatry. 2018;83:840.

12. Langfelder $P$, Horvath S. WGCNA: an R package for weighted correlation network analysis. BMC Bioinformatics. 2008;9:559.

13. Soria-Valles C, Gutierrez-Fernandez A, Osorio FG, Carrero D, Ferrando AA

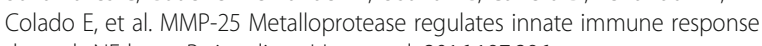
through NF-kappaB signaling. J Immunol. 2016;197:296.

14. Bam M, Yang X, Zumbrun EE, Zhong Y, Zhou J, Ginsberg JP, et al. Dysregulated immune system networks in war veterans with PTSD is an outcome of altered miRNA expression and DNA methylation. Sci Rep. 2016; 6:31209.

\section{Publisher's Note}

Springer Nature remains neutral with regard to jurisdictional claims in published maps and institutional affiliations.

Ready to submit your research? Choose BMC and benefit from:

- fast, convenient online submission

- thorough peer review by experienced researchers in your field

- rapid publication on acceptance

- support for research data, including large and complex data types

- gold Open Access which fosters wider collaboration and increased citations

- maximum visibility for your research: over $100 \mathrm{M}$ website views per year

At $\mathrm{BMC}$, research is always in progress.

Learn more biomedcentral.com/submissions 